\title{
Politique
}

Politique

\section{Mabel Tinkiss Good, Lionel Chevrier, Montréal, Stanké, 1987, 251 p. (Traduit de l’anglais par Michelle Tisseyre)}

\section{Michel Sarra-Bournet}

Numéro 12, automne 1987

Mouvements et acteurs

URI : https://id.erudit.org/iderudit/040578ar

DOI : https://doi.org/10.7202/040578ar

Aller au sommaire du numéro

Éditeur(s)

Société québécoise de science politique

ISSN

0711-608X (imprimé)

1918-6584 (numérique)

Découvrir la revue

Citer ce compte rendu

Sarra-Bournet, M. (1987). Compte rendu de [Mabel Tinkiss Good, Lionel Chevrier, Montréal, Stanké, 1987, 251 p. (Traduit de l'anglais par Michelle

Tisseyre)]. Politique, (12), 154-159. https://doi.org/10.7202/040578ar d'utilisation que vous pouvez consulter en ligne.

https://apropos.erudit.org/fr/usagers/politique-dutilisation/ 
Mabel Tinkiss Good, Lionel Chevier, Montréal, Stanké, 1987, 251 p. (Traduit de l'anglais par Michelle Tisseyre).

La biographie politique est un art difficile, surtout lorsqu'il s'agit, de surcroît, de biographie historique. C'est un monde dans lequel on doit entrer préparé. L'auteure, enseignante, journaliste, écrivaine et, sur la fin de sa carrière, «spécialiste de l'histoire Canadienne et du folklore», se dit peu intéressée à la politique. Elle est attirée par l'époque et par le fait que la carrière de Chevrier embrasse plusieurs domaines. Voici un agenda intéressant. Malheureusement, la signifiance du personnage est principalement politique, puisqu'il a été député et ministre de 1935 à 1954 et 
de 1957 à 1964, puis diplomate de 1964 à 1967 , en 1968 et en 1970. Chevrier n'œuvre à plein temps que neuf ans dans les «autres domaines». Par conséquent, le sujet force l'auteure à traiter de politique la grande majorité du temps, à l'exception des chapitres sur sa jeunesse et ses années de formation. En fait, seulement quarante pages sont consacrées à son passage à l'Administration de la Voie maritime du St-Laurent entre 1954 et 1957. À peine un paragraphe décrit ses activités juridiques à Montréal en 1969 et après 1970 .

Tout au long du livre, on se retrouve donc devant une série d'occasions ratées d'expliquer la présence de ce Franco-Ontarien aux premières loges de la vie politique canadienne et les pratiques politiques de son époque. Nous en relaterons quelques-unes tout en retraçant la carrière de Chevrier.

Les parents de Lionel Chevrier étaient originaires de l'Ouest de Montréal. Ils ont déménagé à Cornwall, dans un secteur à majorité anglophone. Lionel fait ses classes sous le Règlement 17, mais il préfere discuter de la Voie maritime, un projet qui hante sa région depuis des temps immémoriaux. Même la religion compte plus que la barrière linguistique. Celle-ci n'existe pas pour lui. Il fait partie des premiers inscrits au Baccalauréat Art et Science à l'Université d'Ottawa. Après avoir abandonné le Grand Séminaire de Montréal parce qu'il n'avait pas la vocation, on le retrouve au Osgoode Hall Law School de Toronto, au grand dam de son père. De 1930 à 1935, il pratique le droit à Cornwall. La grande question à laquelle on ne répond pas est «Pourquoi la politique?». Outre les discussions qu'il écoute distraitement, entre son père et d'autres, rien ne semble expliquer sa présence à l'assemblée libérale de nomination dans Stormont, en 1935. Quelques hypothèses sur sa socialisation politique auraient pu être émises ou, à la rigueur, l'opinion du principal intéressé lui-même, qui était toujours vivant au moment de la recherche. 
Les années de Chevrier comme simple député (de 1935 à 1943) sont à peine esquissées. La discussion du contexte politique est décevante. On confond CCF, UFA et Crédit Social. L'analyse de l'élection de 1935 est faible. On résume 20 ans en trois lignes, tout en se trompant sur le temps écoulé depuis la Grande Guerre: «Les choses bougeaient après 12 années déchirées par la guerre, les excès qui l'avaient suivie et la longue crise économique.» (p. 85). Outre un bon mais court paragraphe sur les ressources des députés de l'époque, on mentionne à peine que des intérêts régionaux faisaient pression sur Chevrier. On parle, sans les citer ni les nommer, des bons souvenirs des hommes d'affaires et des professionnels de Stormont qui ont retenu l'attention de Chevrier. On effleure la vie du caucus au passage, on parle des tragédies de la famille royale, on fait l'apologie des réalisations de la première session (ou était-ce la deuxième?) du Gouvernement King, on parle des quelques discours de Chevrier en Chambre en 1937 et 1939, puis on saute directement à 1943. Cette année-là, Chevrier, de même que Paul Martin de Windsor, élu comme lui huit ans plus tôt, est nommé secrétaire parlementaire. On aurait pu élaborer sur cette fonction nouvelle dans la gestion du gouvernement canadien.

Avant les élections de 1945, Chevrier est appelé au Cabinet. Il préfere le portefeuille des Transports à celui du Revenu national (pourquoi?). Pas un mot sur la coutume de choisir un nouveau cabinet immédiatement avant une élection générale, plutôt qu'après. Les années de Chevrier comme ministre semblent avoir passé très vite. On s'attarde un peu sur l'historique du ministère, sur son personnel de hauts fonctionnaires et sur l'importance grandissante des transports au Canada, mais on omet de faire un bilan des réalisations de Chevrier en 9 ans, à deux exemples près: le chemin de fer de Kitimat, Colombie-Britannique et le chemin de Canso, à l'Île du Cap Breton. Très peu de détails sur les négociations 
qui y ont conduit, pas du tout sur les tractations en coulisses, car il a dû y en avoir. Le rôle de Chevrier dans la course au leadership de 1948 où, lui-même candidat, il a transféré ses appuis à St-Laurent, reste obscur. La même année, sa présence à l'assemblée générale de l'ONU apparaît figurative. En 1954, le premier ministre lui offre la présidence de l'Administration de la Voie maritime du Canada.

Chevrier accepte, ce qui est en accord avec le vieux rêve qui avait accompagné son enfance. Mais les éléments fournis par l'auteure établissent qu'il s'est réalisé grâce à la volonté politique de StLaurent. De son propre aveu, la qualité de Chevrier semble d'avoir été au bon endroit au bon moment. L'auteure, bien qu'elle parle d'un "grand Canadien, totalement dévoué à la cause d'un Canada uni» (p. 29), nous présente, finalement un personnage qui sert d'instrument entre les mains d'acteurs plus significatifs, ou de médium par lequel le destin a façonné le Canada. Il est difficile de croire que Chevrier ait été si passif devant les circonstances, que sa seule contribution à l'Histoire ait été de faire ajouter une statue de Montcalm à celle de Wolfe, devant la Maison du Canada à Londres, lorsqu'il était Haut-Commissaire dans les années 1960.

Une fois le défi de la Voie maritime relevé, Chevrier répond à l'appel d'un St-Laurent en difficulté, et se fait réélire dans une circonscription montréalaise, en 1957. Les déboires des Libéraux font en sorte qu'il devient une des quatre figures dominantes de l'Opposition jusqu'à 1963, avec Pickersgill, Martin et Pearson à ses côtés. Son appui à ce dernier lors du congrès au leadership de 1958 lui vaudra de la reconnaissance qui ne tardera pas à rapporter. Lorsque lassé du va-et-vient dans son bureau de Ministre de la Justice en 1963-64, il décide qu'il n'a plus le feu sacré de la politique, Pearson lui offre un poste d'ambassadeur. Chevrier préfere Londres à Paris, sur les conseils «d'amis» de Cornwall qui le mettent en garde contre la personnalité du Général de 
Gaulle! Sa participation aux conférences du Commonwealth lui donnera une expérience de l'Afrique. La suite vient renforcer l'impression qu'il est l'homme «du bon moment, au bon endroit». Son expérience de la diplomatie l'amène à être Commissaire général des visites d'État d'Expo 67. À cette occasion, de Gaulle lui aurait expliqué la signification de son «Vive le Québec libre». Malheureusement, Chevrier ne l'a pas confiée à l'auteure. On devra attendre de mettre la main sur son mémo au Ministère des Affaires extérieures. En 1968, grâce à ses connaissances de la politique africaine, on a fait appel à lui pour une tournée du continent afin de préparer la politique de coopération du Canada. En 1969, il pratique le droit à Montréal. En 1970, il fait une tournée de promotion aux États-Unis, au nom des Affaires extérieures, sans trop de succès. Il termine sa carrière dans la pratique du droit.

Lionel Cheurier décrit les méandres d'une carrière politique à la manière de mémoires politiques. Il est à soupçonner que cet ouvrage a été écrit à partir d'interviews avec Chevrier, bien qu'on n'ait que des indices à ce sujet. En effet, pas de références précises, lorsqu'il y en a, ni de biblographie susceptible d'aider ceux ou celles qui désireraient poursuivre cette étude. Tout au plus quelques allusions au journal et à la correspondance de Mackenzie King et à des journaux portent à croire qu'il s'agit plus que les mémoires de Lionel Chevrier écrites par quelqu'un d'autre. Cependant, l'origine de l'auteure, qui a grandi dans la même ville, fréquenté les mêmes lieux, connu Chevrier et sa famille font qu'elle était trop proche de lui pour être objective. Par conséquent, on lit un ouvrage qui respire l'optimisme et le progrès. On en retient toutefois l'image d'un homme qui était plus spectateur qu'acteur, la courroie de transmission de ses chefs politiques, en quelque sorte. Son apport à la cause du français au Canada est en droite ligne avec l'école du bilinguisme, du dualisme et de l'unité. 
(L'auteure parle du "cri du balcon» comme d'un incident malheureux). En général, le traitement du contexte socio-politique est superficiel. On s'arrête plus volontiers sur la personnalité des individus, sur des anecdotes et sur la petite histoire. Bref, un ouvrage plus impressionniste qu'analytique ou même descriptif. Au cours des dernières décennies, la tendance en biographie historique était de dépasser l'individu pour analyser son époque. Ici, malgré les bonnes intentions, on n'y arrive pas, pas plus qu'on n'atteint les motivations de l'individu même. L'ouvrage de $M$. T. Good n'est définitivement pas un modèle dans le genre biographique.

Michel Sarra-Bournet

Université d'Ottawa 